IMECE2016-67509

\title{
Influencing factors and scaling law of bubble detachment at a submerged orifice
}

\author{
Yiwei Wang \\ Key Laboratory for Mechanics in \\ Fluid Solid Coupling Systems, \\ Institute of Mechanics, Chinese \\ Academy of Sciences \\ Beijing, China
}

\author{
Chenguang Huang \\ Key Laboratory for Mechanics in \\ Fluid Solid Coupling Systems, \\ Institute of Mechanics, Chinese \\ Academy of Sciences \\ Beijing, China
}

\author{
Xiaocui Wu \\ Key Laboratory for Mechanics in \\ Fluid Solid Coupling Systems, \\ Institute of Mechanics, Chinese \\ Academy of Sciences \\ Beijing, China
}

\begin{abstract}
Bubble formation and detachment are important in the drag reduction of underwater vehicle by using gas injection, which are involved in the integrated scaling law considering bubbles coalescence. The parameters of influencing factors are theoretically derived by dimensionless analysis on this issue. Then the effects are shown by analyzing results with various parameters. Results indicate that, the viscous force can be neglected near the default condition, and the bubble is difficult to detach if the viscosity is very large. Surface tension is the major constraint force in the default condition, which is also sensitive. The bubble easily crushes if the surface tension is small, while the neck of bubble can hardly break up if the surface tension is large. For the contact angle, the bubble profile significantly exceeds the orifice boundary if the wall surface is hydrophobic, which makes detachment period becomes larger.
\end{abstract}

\section{INTRODUCTION}

Bubble formation and detachment are involved in many engineering applications. Owning to its practical importance, the phenomenon of bubble formation and pitch-off has been widely studied theoretically and experimentally for decades [113]. As new application directions, bubble drag reduction (BDR) and air-layer drag reduction (ALDR) are important methods to reduce the Skin-friction drag of high speed underwater vehicles [14-17]. For the external multiphase flow field around underwater vehicles, the major similarity parameters include Froude number, cavitation number and Euler number. However, on the issue of bubble and air layer formation, firstly the gas ejects from the orifice as the form of discrete bubbles, and then the bubbles coalesce into continuous air layer. Coalescence is the key process of flow pattern transition of drag reduction, because the effect of ALDR is more significant than BDR [14].

However besides the influencing factors of the external flow, the flow pattern may be closely related to the flow field and bubble state near the orifice. Therefore this requires indepth study on the microscopic flow filed of bubble formation and detachment, especially the dynamic characteristics. We need to obtain the influencing factors and the scaling laws, which can be combined with the factors of the macroscopic external flow to derive the integrated scaling law of bubbles coalescence and ALDR.

In the present paper, numerical and experimental methods on bubble formation and detachment are established. The parameters of influencing factors are theoretically derived by dimensionless analysis. Then the effects are shown by analyzing results with various parameters

\section{EXPERIMENTAL AND NUMERICAL METHODS}

The experiment of bubble formation on submerged orifice was conducted at atmospheric pressure and temperature. Experiment setup is sketched in Fig.1. A glass tank with stainless steel plate at the bottom was used. The cross section of the tank was $500 \mathrm{~mm} \times 500 \mathrm{~mm}$ with an orifice in the middle of the plate. The diameter of the orifice was $2 \mathrm{~mm}$. Compressed air was forced to the orifice through a long plastic tube and retaining valve. A flow rate controller was used to control the gas flow rate. In experiment of present work, the depth of water in the glass tank was $100 \mathrm{~mm}$ and the gas flow rate was $100 \mathrm{ml} / \mathrm{min}$. The high-speed camera is placed on the side of the tank, above and parallel to the bottom plate. 


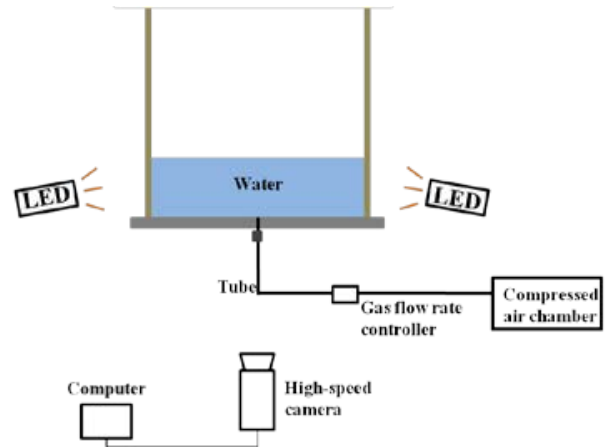

Fig.1 Sketch of experiment setup

The process of bubble formation was recorded by a highspeed camera. The bubble formation frequency and bubble morphology could be observed by recorded movie files, as shown in Fig.2. With a frame grabbing speed of 6000 frames/s, the bubble periods could be measured with an accuracy of $\pm 1 / 6 \mathrm{~ms}$. The experiment results were compared with numerical ones in following part.

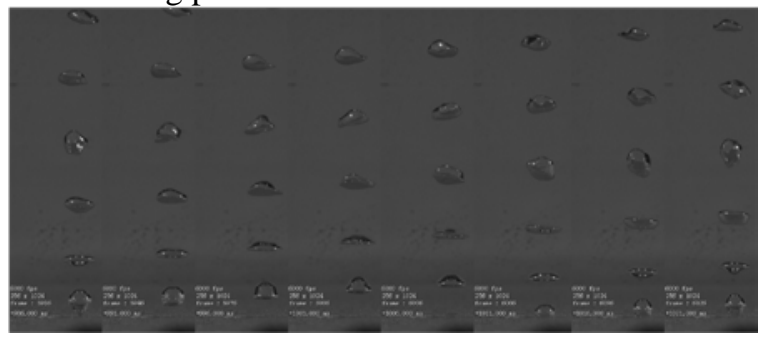

Fig.2 Bubble pictures at gas flow rate of $100 \mathrm{ml} / \mathrm{s}$ in experiment (The time interval is $5 \mathrm{~ms}$.)

For the bubble formation as a two phase flow phenomenon, an implicit large eddy simulation (ILES) method is adopted and combined with a volume of fluid (VOF) scheme.

The Navier-Stokes equations of incompressible flow by applying the filter function of LES are as follows:

$$
\begin{aligned}
& \nabla \bullet(\rho \overline{\mathbf{v}})=0 \\
& \frac{\partial}{\partial \mathrm{t}}(\rho \overline{\mathbf{v}})+\nabla \bullet(\rho \overline{\mathbf{v}} \overline{\mathbf{v}})=-\nabla \bar{p}+\nabla \cdot(\overline{\mathbf{S}}-\mathbf{B})
\end{aligned}
$$

where the over-bar denotes the low-pass filtered dependent variables as $\bar{\psi}=\int_{-\infty}^{+\infty} \psi G\left(x, x^{\prime}\right) d x^{\prime} . G\left(x, x^{\prime}\right)$ is the filter function, where top-hat filter function is adopted in this paper.

$$
\overline{\mathbf{S}}=2 \mu \overline{\mathbf{D}} \text { is the filtered viscous stress tensor, }
$$
$\overline{\mathbf{D}}=\frac{1}{2}\left(\nabla \overline{\mathbf{v}}+\nabla \mathbf{v}^{T}\right)$ stands for the filtered rate of stress tensor and $\mu$ is the dynamic viscosity. $\mathbf{B}=(\overline{\mathbf{v v}}-\overline{\mathbf{v} v})$ means the subgrid stress tensor, representing the influence of the small, unresolved eddies on the larger, resolved ones. $\mathbf{B}$ can be decomposed as $\mathbf{B}=(\overline{\overline{\mathbf{v}}}-\overline{\overline{\mathbf{v}}} \overline{\mathbf{v}}+\tilde{\mathbf{B}})$. The ILES is adopted in the present method where no SGS model is implemented for $\tilde{\mathbf{B}}$. The unresolved turbulent motions are assumed to be mimicked by the numerical effects of discretization[18, 19].

For the VOF model, additional transport equation for the liquid volume fraction $\alpha$ is solved as

$$
\frac{\partial \alpha}{\partial t}+\nabla \bullet(\overline{\mathbf{v}} \alpha)=0
$$

Then the density and viscosity in the Navier-Stokes equations should be replace by the mixture values as

$$
\begin{aligned}
& \rho=\alpha \rho_{l}+(1-\alpha) \rho_{g} \\
& \mu=\alpha \mu_{l}+(1-\alpha) \mu_{g}
\end{aligned}
$$

where subscripts $l$ and $g$ denote the liquid and gas phases, respectively.

The interface is reconstructed using the algebraic solver. a counter gradient transport method[20, 21] is used to advect the interface and limit the numerical diffusion. Therefore, we can determine the pinch-off process by whether the interfaces converge at the neck of the bubble.The present method is established under the framework of the open source code OpenFOAM with the solver interFoam.

An axisymmetric solution domain was adopted in this paper (as shown in Fig.3). The diameter of the axisymmetric solution domain $\mathrm{R}$ is set as $50 \mathrm{~mm}$ to ensure that the bubble formation process is not affected by the side wall. In the default condition, the orifice is set as flow rate boundary with $Q=100 \mathrm{ml} / \mathrm{min}$ and radius $r_{o}=1 \mathrm{~mm}$. Length of the channel $\mathrm{l}$ is $4 \mathrm{~mm}$. The top surface of the computation domain is set as a pressure outlet boundary and all the walls are set as non-slip wall. A constant contact angle condition is adopted. The contact angle is defined as the angle between the surface of the liquid and the outline of the contact surface. Between $0^{\circ}$ and $90^{\circ}$, the solid is wettable and above $90^{\circ}$ it is not wettable. First order implicit Euler time scheme and second order Gauss liner interpolation in space are adopted.

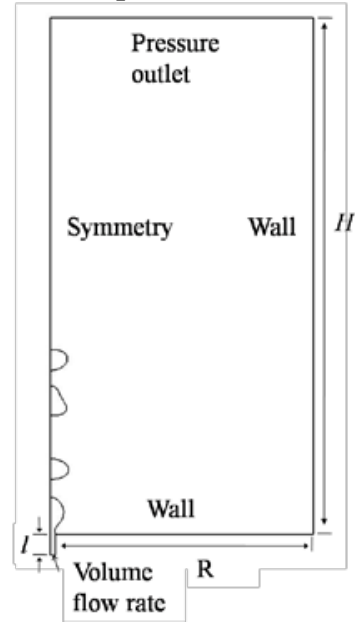

Fig.3 Simulation domain and boundary conditions

Three grids are used for the independence study. The cell numbers and maximum lengths of the bubbles at the pinch-off 
moments are list in Tab.1, and the bubble shapes are compared in Fig.4. Results indicate that the grid number is enough for the medium mesh, which is used for the following analysis.

Tab.1 Results of grid independence study

\begin{tabular}{|c|c|c|}
\hline & $\begin{array}{c}\text { Cell } \\
\text { numbers }\end{array}$ & $\begin{array}{c}\text { Maximum } \\
\text { lengths (mm) }\end{array}$ \\
\hline Coarse mesh & $3.6 \mathrm{E} 4$ & 11.82 \\
\hline Medium mesh & $1.4 \mathrm{E} 5$ & 11.80 \\
\hline Fine mesh & $5.6 \mathrm{E} 5$ & 11.80 \\
\hline
\end{tabular}
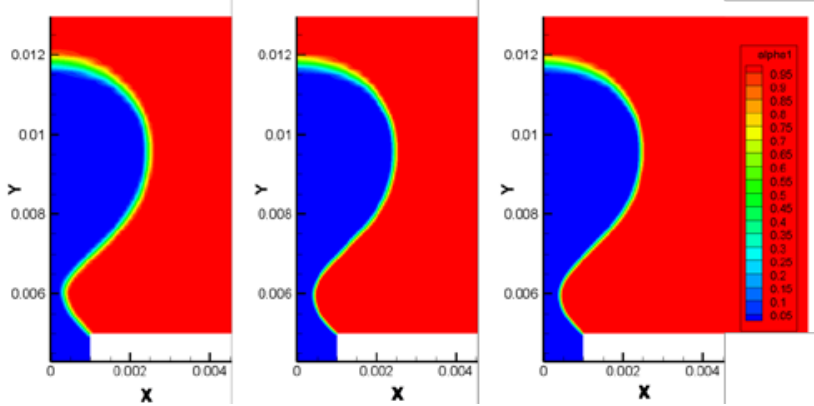

Fig.4 Comparisons of bubble shapes in grid independence study. (The left, middle and right views are results with coarse, medium and fine meshes, respectively.)

\section{THEORETICAL ANALYSIS ON THE SIMILARITY PARAMETERS}

Under the default condition, bubbles form and detach periodically. The detachment frequency and the maximum diameter are major investigated. The governing parameters are listed as following.

$$
d_{\max }=f\left(\rho, \mu, \gamma, \delta, v, p_{\infty}, H, g, \alpha\right)
$$

Where $d_{\max }$ is the maximum diameter of the bubble before detachment, $\rho$ and $\mu$ are the density and viscosity coefficient of liquid water, respectively. $\gamma$ is the surface tension coefficient. $\delta$ is the diameter of the orifice. $v$ is the velocity of gas injection. $p_{\infty}$ is the background pressure of the far field. $H$ is the water depth, $g$ is the gravity acceleration. $\alpha$ is the contact angle of the wall. Because the density ratio between water and gas is large, the flow is dominated by the liquid water, and the density and viscosity of gas can be neglected.

The formula is nondimensionalized as following by selecting $\rho, g$ and $\delta$ as the basic quantities.

$$
\frac{d_{\max }}{\delta}=f\left(\frac{\rho g^{\frac{1}{2}} \delta^{\frac{3}{2}}}{\mu}, \frac{\rho g \delta^{2}}{\gamma}, \frac{\sqrt{g \delta}}{v}, \frac{H}{\delta}, \alpha\right)
$$

where $\frac{\rho g^{\frac{1}{2}} \delta^{\frac{3}{2}}}{\mu}$ represents the ratio of buoyancy and viscous forces, $\frac{\sqrt{g \delta}}{v}$ represents the ratio of buoyancy and inertia forces, $\frac{\rho g \delta^{2}}{\gamma}$ represents the ratio of buoyancy and surface tension forces, which is also called Bond number or Eötvös number, $\frac{H}{\delta}$ is the dimensionless depth.

For the scaling law, considering $\lambda$ as the length scale factor as $\frac{\delta_{p}}{\delta_{m}}=\lambda$, where subscripts $p$ and $m$ represent the practice and model, respectively. The density $\rho$ and gravity acceleration $g$ are constant with various scales. The depth should similar as a geometry value, and the contact angle should be constant as the surface property. The relationships can be derived as

$$
\frac{\delta_{p}}{\delta_{m}}=\lambda, \frac{g_{p}}{g_{m}}=1, \frac{\rho_{p}}{\rho_{m}}=1, \frac{H_{p}}{H_{m}}=\lambda, \frac{\alpha_{p}}{\alpha_{m}}=1,
$$

Therefore, the integrated scaling law is as following.

$$
\frac{\mu_{p}}{\mu_{m}}=\lambda^{\frac{3}{2}}, \frac{\gamma_{p}}{\gamma_{m}}=\lambda^{2}, \frac{v_{p}}{v_{m}}=\sqrt{\lambda}
$$

And we can further derive the dependent variables satisfy the relationship as

$$
\frac{d_{\max p}}{d_{\max m}}=\lambda, \frac{T_{p}}{T_{m}}=\sqrt{\lambda}
$$

where $T$ is the period of bubble detachment.

Aforementioned equations compose the similarity law the present issue.

\section{VALIDATIONS OF THEORETICAL AND NUMERICAL METHODS}

Periodical detachment phenomena in the default condition can be also found in typical experiments in the reference [1] (as shown in Fig.5), and the period of detachment is $46 \mathrm{~ms}$.

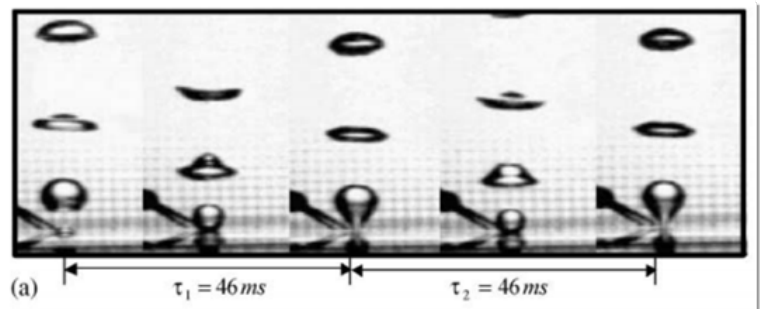

Fig.5 Experimental results in the default condition in reference[1]

Simulation in the same condition is performed and the results agree well with the experimental results (as shown in the Fig. 6). The difference between the periods is less than $3 \%$ 。 


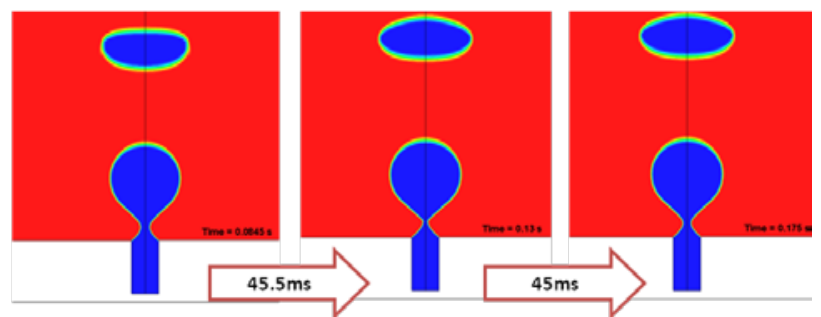

Fig.6 Numerical simulation results in the default condition

On this basis, we can validate the similarity law. $\lambda=4$ is selected. All the dimensionless parameters are kept unchanged by varying the material properties. Therefore the results should satisfy

Results are shown as Fig.6. Compared to the default condition, the bubble shapes are similar and the maximum diameter also scales as eqn(10). We can also find the period is twice as the default case which also meet the relationship as $\sqrt{\lambda}$ of the theoretical results.

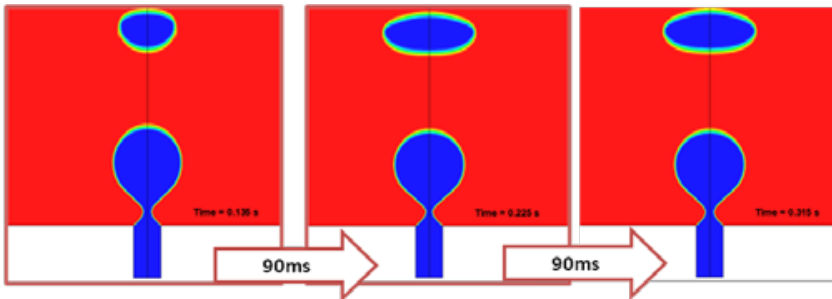

Fig.7 Verification results of the similarity law after amplifying 4 times as the default condition

\section{EFFECTS OF THE INFLUENCING FACTORS}

As shown by the equation (7), the present issue has major influencing factors including buoyancy force, viscous force, surface tension, inertia force of gas injection, dimensionless depth and contact angle. The buoyancy force and inertia force are major active factors of bubble detachment, while the surface tension and viscous force are the major factors which prevent the bubble detachment. The dimensionless depth controls the far field condition, and the contact angle is the constraint geometrical condition of the wall surface. The bubble becomes larger before detachment if the period is longer. Therefore, the effects of the influencing factors are investigated by varying the parameters of a default condition (as list in Tab.2) while maintaining the buoyancy force. Therefore the density and gravity acceleration are kept unchanged.

Tab.2 Simulation parameters of the default condition

\begin{tabular}{|c|c|}
\hline Liquid density $\rho$ & $998.2 \mathrm{~kg} / \mathrm{m}^{3}$ \\
\hline $\begin{array}{c}\text { Gravity acceleration } \\
g\end{array}$ & $9.81 \mathrm{~m} / \mathrm{s}^{2}$ \\
\hline Liquid viscosity $\mu$ & $0.001 \mathrm{~kg} / \mathrm{m}-\mathrm{s}$ \\
\hline $\begin{array}{c}\text { Surface tension } \\
\text { coefficient } \gamma\end{array}$ & $0.07 \mathrm{~N} / \mathrm{m}$ \\
\hline Diameter of the orifice & $2 \mathrm{~mm}$ \\
\hline
\end{tabular}

\begin{tabular}{|c|c|}
\hline$\delta$ & \\
\hline $\begin{array}{c}\text { Volume flow rate of gas } \\
\text { injection } Q\end{array}$ & $100 \mathrm{ml} / \mathrm{min}$ \\
\hline Depth of the orifice $H$ & $100 \mathrm{~mm}$ \\
\hline Contact angle $\alpha$ & $60^{\circ}$ \\
\hline
\end{tabular}

\subsection{Effect of viscosity}

The viscosity prevents the acceleration and detachment of the bubble as a drag force. $\left.\frac{\rho g^{\frac{1}{2}} \delta^{\frac{3}{2}}}{\mu}\right\rangle_{1}$ in the default condition which demonstrate the viscous force is much less than the buoyancy force. Therefore the variation of viscosity in a small range won't affect the result.

Bubble formation and detachment with the viscosity is amplified as 10, 100, 1000 and 10000 times are shown in Fig.8. The period and shapes are the same as the default case when the viscosity is amplified as 10 times (as shown in Fig.8-a). The period and shapes are slightly larger when the viscosity is amplified as 100 times (as shown in Fig.8-b). Bubbles cannot detach clearly when the viscosities are amplified as 100 and 1000 times (as shown in Fig.8-c and 8-d) because the viscous forces are larger than the buoyancy force under these conditions.
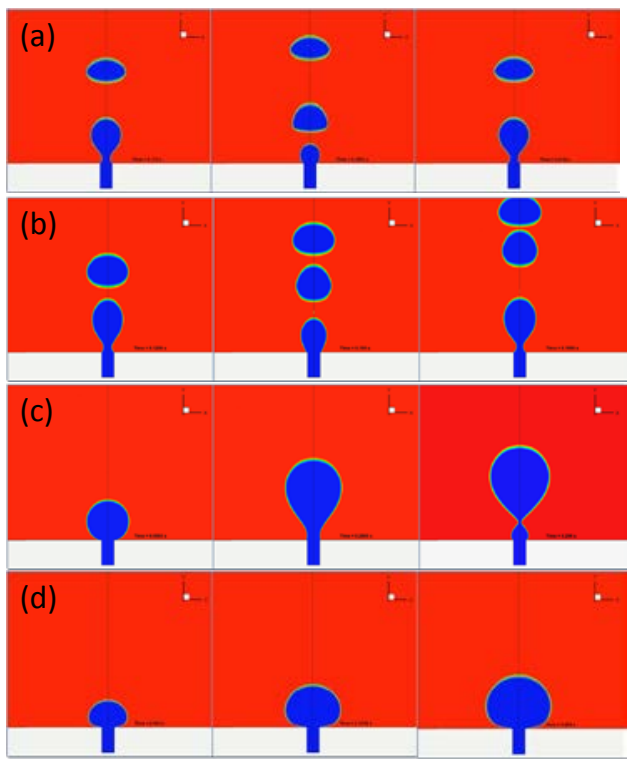

Fig. 8 Bubble evolutions when varying the viscosity (a: 10 times, b: 100 times, c: 1000 times, d: 10000 times)

Periods of bubbles detachments and the maximum bubble diameters are shown in Tab.3, which are similar near the default condition. However bubbles are difficult to detach if the viscosity is very large.

Tab.3 Time periods and maximum diameters of bubbles under various viscosities

\begin{tabular}{|c|c|c|}
\hline $\begin{array}{c}\text { Amplification } \\
\text { factors }\end{array}$ & Period (ms) & $\begin{array}{c}\text { Max diameter } \\
(\mathrm{mm})\end{array}$ \\
\hline Default condition & 45.0 & 4.98 \\
\hline
\end{tabular}




\begin{tabular}{|c|c|c|}
\hline 10 & 45.0 & 5.03 \\
\hline 100 & 57.0 & 5.31 \\
\hline 1000 & N/A & 9.48 \\
\hline 10000 & N/A & N/A \\
\hline
\end{tabular}

\subsection{Effect of surface tension}

The value of surface tension force is close to the buoyancy force under the default condition, which may has important effect as the major constraint force. The effect of surface tension is represented by the dimensionless number as Bond number or Eötvös number.

Bubble evolutions when the surface tension is reduced to $1 \%$ of the default case are as shown in Fig. 9-a. Because surface tension is the key factor to maintain the spherical shape of the bubble, the critical diameter of forming spherical bubble is smaller than the diameter of the orifice in this case. Therefore the bubble volume and detachment period both become very small, and the bubbles are easy to break up. If the surface tension is amplified to 10 times as the default case, the bubble can hardly detach under the strong constraint force of surface tension (as shown in Fig.9-b). The bubble diameter is about 2 times of the default case, and the bubble oscillates and sticks to the orifice. The time periods and maximum diameters of the bubble are list in Tab.4.
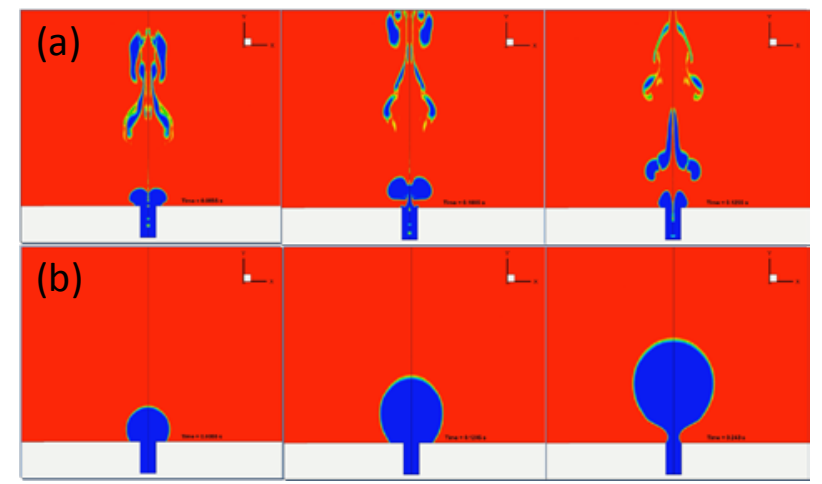

Fig. 9 Bubble evolutions when varying the surface tension (a:1\%, b:10 times)

Tab.4 Time periods and maximum diameters of bubbles under various surface tension coefficients

\begin{tabular}{|c|c|c|}
\hline $\begin{array}{c}\text { Amplification } \\
\text { factors }\end{array}$ & Period (ms) & $\begin{array}{c}\text { Maximum } \\
\text { diameter (mm) }\end{array}$ \\
\hline $\begin{array}{c}\text { Default } \\
\text { condition }\end{array}$ & 45.0 & 4.98 \\
\hline 0.01 & N/A & N/A \\
\hline 0.1 & 19.3 & 4.17 \\
\hline 10 & N/A & 10.1 \\
\hline
\end{tabular}

\subsection{Effect of the inertia force}

Inertia force is an important factor on bubble growth and detachment. Gas flow inside the bubble can push the bubble interface upwards and accelerate the bubble growth and detachment. Moreover, the wake flow around the detached bubbles can also affect the interface movement of the following bubble (the typical flow field is as shown in Fig.10).

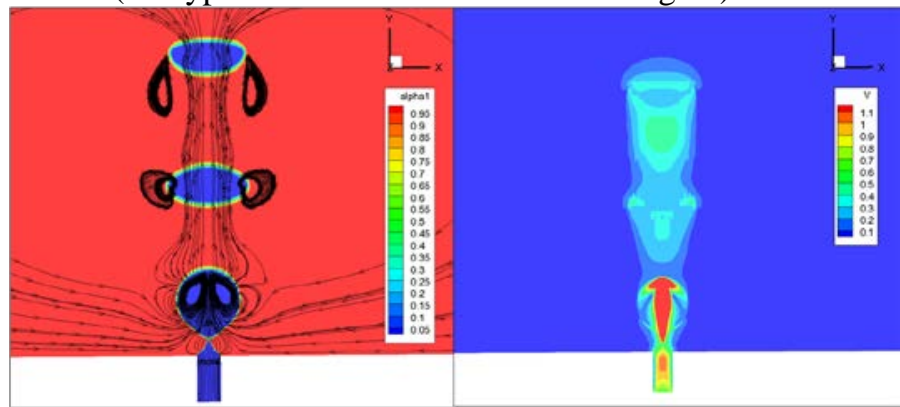

Fig. 10 Streamlines and velocity magnitude contours at the moment of bubble pinch-off in the default case

The effect of the inertia force can be analyzed by varying the flow rate. There are several regimes if increasing the flow rate. Firstly, the paring model emerges when bubbles coalesce with each other after detachment (as shown in Fig.11), which can affect the growth and detachment processes of the next bubble. Moreover, double and triple coalescences take place if the flow rate is large, bubbles coalesce into larger bubbles before detachment, and adjacent cycles have quite different time periods (as shown in Fig.12). These effects are also well investigated in Zhang et al 's work [1].

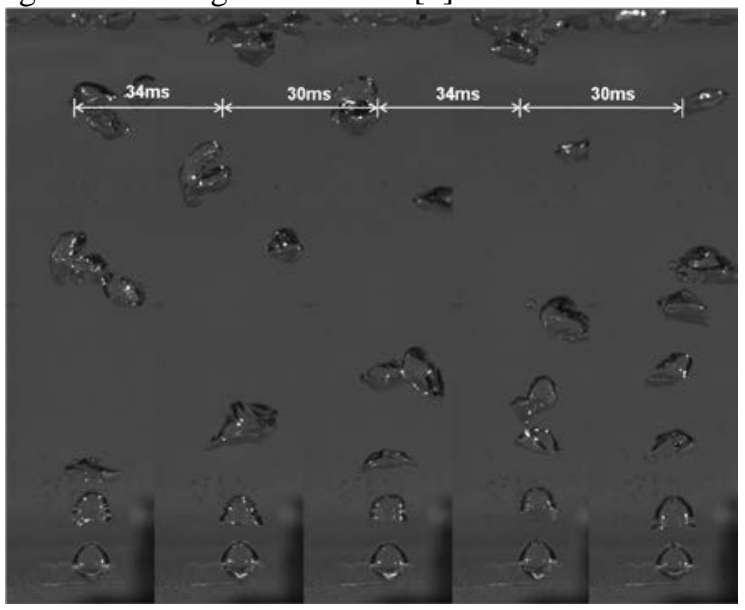

Fig. 11 Bubble evolutions when the flow rate is $350 \mathrm{ml} / \mathrm{min}$. (There are two different time periods because of bubbles coalescence after detachment) 


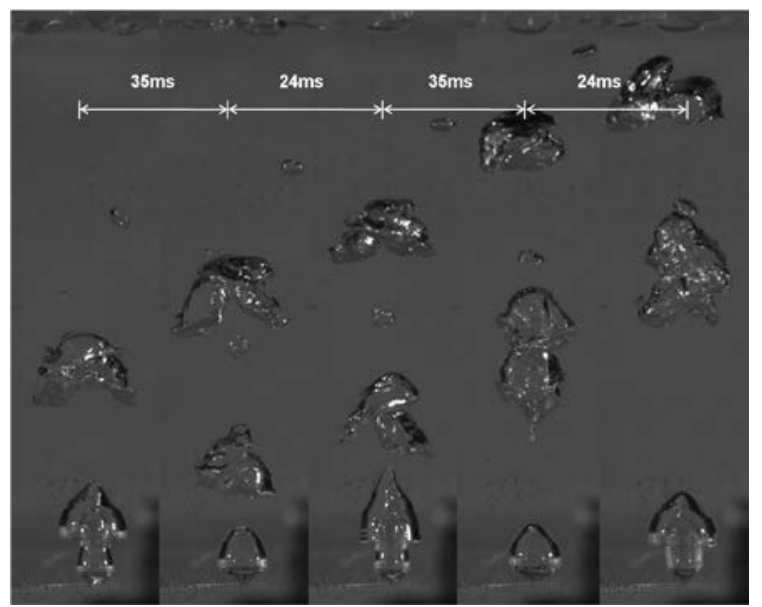

Fig. 12 Bubble evolutions when the flow rate is $900 \mathrm{ml} / \mathrm{min}$. (There are two different time periods because of bubbles coalescence before detachment)

\subsection{Effect of dimensionless depth}

Because the dimensionless depth $\frac{H}{\delta}$ is much larger than 1 , the effect is small if the value is only changed within a small range near the default condition.

The bubble evolution is as shown in Fig.13 when the depth is changed to half of the default case. The period is unchanged as $45 \mathrm{~ms}$ and the bubble shape is also similar with the default case. Therefore, the depth doesn't affect the bubble formation and detachment if the free surface is far enough from the orifice. The time periods and maximum diameters of the bubble are list in Tab.5.

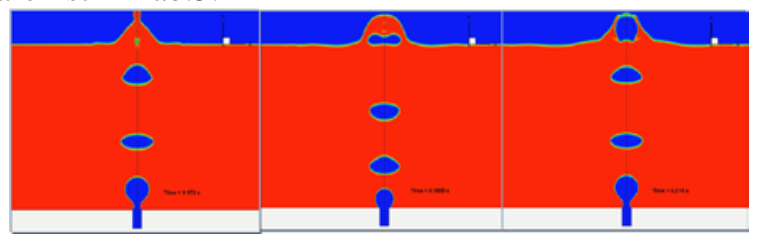

Fig. 13 Bubble evolutions when depth is changed to half of the default case

Tab.5 Time periods and maximum diameters of bubbles under various submerged depth

\begin{tabular}{|c|c|c|}
\hline $\begin{array}{c}\text { Amplification } \\
\text { factors }\end{array}$ & Period (ms) & $\begin{array}{c}\text { Maximum } \\
\text { diameter (mm) }\end{array}$ \\
\hline $\begin{array}{c}\text { Default } \\
\text { condition }\end{array}$ & 45.0 & 4.98 \\
\hline 0.4 & 45.0 & 4.99 \\
\hline 0.6 & 45.0 & 5.00 \\
\hline 0.8 & 45.0 & 4.97 \\
\hline
\end{tabular}

\subsection{Effect of contact angle}

The effect of the contact angle is complicated which majorly affects the geometrical constraint condition between the interfaces. The contact angle is $60^{\circ}$ in the default case.
Bubble evolutions with various contact angles are shown in Fig.14. The liquid water strongly wets the wall under small contact angles, and the bubble shapes and detachment periods are similar (as shown in Fig.14-a \& b). However, the lower edge of the bubble exceeds the boundary of the orifice and stick to the bottom surface if the contact angle is larger than $60^{\circ}$, in which case the buoyancy force actually decrease in the expansion stage. So the period becomes larger (as shown in Fig.14-c \& d).

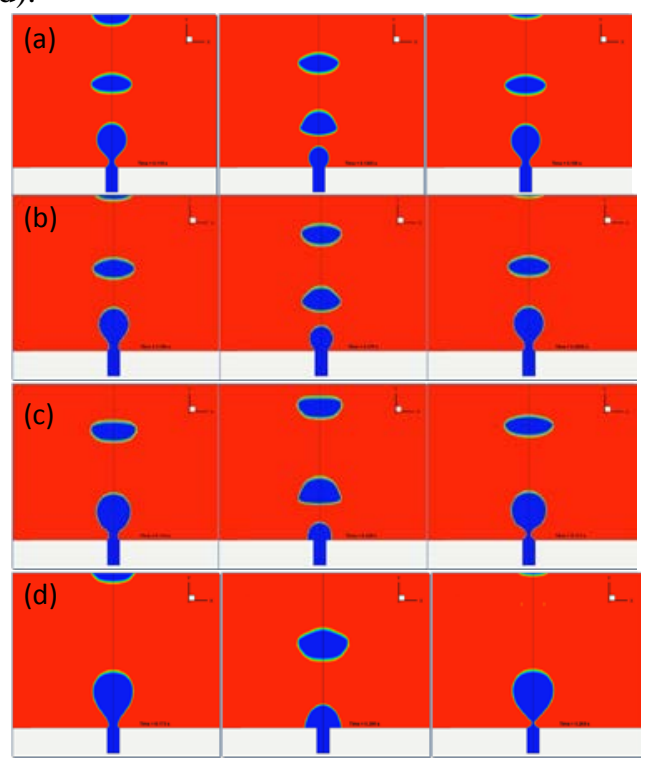

Fig. 14 Bubble evolutions when varying the contact angle (a: $20^{\circ}$, b: $40^{\circ}$, c: $80^{\circ}$, d: $100^{\circ}$ )

The detachment periods and maximum parameters under various conditions are list in Tab.6. Results demonstrate that the value is approximately constant if the contact angle is small, while the period and maximum diameter become larger if the contact angle increases. If the wall surface is superhydrophobic, the bubble profile significantly exceeds the orifice boundary and the projected area of the bubble on the bottom surface becomes larger, which may make the bubbles closer to each other and the coalescence become easier.

Tab.6 Time periods and maximum diameters of bubbles under various contact angles

\begin{tabular}{|c|c|c|}
\hline contact angle $\left(^{\circ}\right)$ & Period (ms) & $\begin{array}{c}\text { Maximum } \\
\text { diameter (mm) }\end{array}$ \\
\hline 20 & 41.5 & 4.79 \\
\hline 40 & 41.5 & 4.78 \\
\hline $\begin{array}{c}60 \text { ( Default } \\
\text { condition ) }\end{array}$ & 45.0 & 4.98 \\
\hline 80 & 58.5 & 5.52 \\
\hline 100 & 89.0 & 6.43 \\
\hline 120 & 116.5 & 8.36 \\
\hline 140 & 133.0 & 9.35 \\
\hline
\end{tabular}




\section{CONCLUSIONS}

The influencing factors on the local flow pattern around the orifice are derived, and the formulas of similarity law are obtained.

The viscous force can be neglected near the default condition, and the bubble is difficult to detach if the viscosity is very large.

Surface tension is the major constraint force in the default condition, which is also sensitive. The bubble easily crushes if the surface tension is small, while the neck of bubble can hardly break up if the surface tension is large.

For the contact angle, the bubble profile significantly exceeds the orifice boundary if the wall surface is hydrophobic, which makes detachment period becomes larger.

Moreover, for the issue of bubble drag reduction, the bubble shape can be affected by the lateral liquid flow stream, which should be further investigated in the future.

\section{ACKNOWLEDGMENTS}

Grateful acknowledgement is made to Dr. Xianxian Yu and Mr. Chao Yu for their help on the data processing of numerical results.

This research was sponsored by National Natural Science Foundation of China under Contract 11332011 and 11202215. This project was also supported by the Youth Innovation Promotion Association CAS (2015015).

\section{REFERENCES}

[1] L. Zhang and M. Shoji, Aperiodic bubble formation from a submerged orifice, Chem. Eng. Sci. 56, 5371 (2001).

[2] D. Legendre, R. Zenit, and J. R. Velez-Cordero, On the deformation of gas bubbles in liquids, Phys. Fluids 24, 043303 (2012).

[3] D. Legendre, On the relation between the drag and the vorticity produced on a clean bubble, Phys. Fluids 19, 018102 (2007).

[4] D. Gerlach, G. Biswas, F. Durst, and V. Kolobaric, Quasistatic bubble formation on submerged orifices, Int. J. Heat Mass Transf. 48, 425 (2005).

[5] V. V. Buwa, D. Gerlach, F. Durst, and E. Schlücker, Numerical simulations of bubble formation on submerged orifices: Period-1 and period-2 bubbling regimes, Chem. Eng. Sci. 62, 7119 (2007).

[6] D. Gerlach, N. Alleborn, V. Buwa, and F. Durst, Numerical simulation of periodic bubble formation at a submerged orifice with constant gas flow rate, Chem. Eng. Sci. 62, 2109 (2007).

[7] A. A. Kulkarni and J. B. Joshi, Bubble formation and bubble rise velocity in gas-liquid systems: A review, Ind. Eng. Chem. Res. 44, 5873 (2005).

[8] A. Marmur and E. Rubin, A theoretical model for bubble formation at an orifice submerged in an inviscid liquid, Chem. Eng. Sci. 31, 453 (1976).

[9] S. Ramakrishnan, R. Kumar, and N. Kuloor, Studies in bubble formation-I bubble formation under constant flow conditions, Chem. Eng. Sci. 24, 731 (1969).
[10] A. Satyanarayan, R. Kumar, and N. Kuloor, Studies in bubble formation-II bubble formation under constant pressure conditions, Chem. Eng. Sci. 24, 749 (1969).

[11] A. Khurana and R. Kumar, Studies in bubble formationIII, Chem. Eng. Sci. 24, 1711 (1969).

[12] K. Terasaka and H. Tsuge, Bubble formation under constant-flow conditions, Chem. Eng. Sci. 48, 3417 (1993).

[13] M. Jamialahmadi, M. Zehtaban, H. Müller-Steinhagen, A. Sarrafi, and J. Smith, Study of bubble formation under constant flow conditions, Chem. Eng. Res. Des. 79, 523 (2001).

[14] B.R. Elbing, E.S. Winkel, K.A. Lay, S.L. Ceccio, D.R. Dowling, M. Perlin, Bubbleinduced skin-friction drag reduction and the abrupt transition to air-layer drag reduction, J. Fluid Mech. 612, 201 (2008).

[15] S.A. Mäkiharju, B.R. Elbing, A. Wiggins, S. Schinast, J.M. Vanden-Broeck, M.Perlin, S.L. Ceccion, On the scaling of air entrainment from a ventilated partial cavity, J. Fluid Mech. 732, 47 (2013).

[16] E.S. Winkel, B.R. Elbing, S.L. CeccioL, M. Perlin, D.R. Dowling, Bubble friction drag reduction in a high-Reynoldsnumber flat-plate turbulent boundary layer, J. Acoust. Soc. Am. 123, 2522 (2008).

[17] X. X. Yu, Y. W. Wang, C. G. Huang, Y. P. Wei. X. Fang, T. Z. Du, X. C. Wu, Experiment and simulation on air layer drag reduction of high-speed underwater axisymmetric projectile, Eur. J. Mech. B-Fluids, 52, 45 (2015).

[18] R. E. Bensow, G. Bark, Implicit LES Predictions of the Cavitating Flow on a Propeller. Journal of Fluids Engineering, 132, 041302(2010).

[19] F. F. Grinstein, L. Margolin, and W. Rider, Implicit Large Eddy Simulation: Computing Turbulent Fluid Dynamics, Cambridge University Press, Cambridge, England (2007). [20] H.G. Welle, The Development of a New Flame Area Combustion Model Using Conditional Averaging. Tech. Rep. TF 9307, Thermo-Fluids Section Report, Imperial College of Science, Technology and Medicine (1993). [21] A. Albadawi, D. B. Donoghue, A. J. Robinson, D. B. Murray, Y. M. C. Delaure, On the analysis of bubble growth and detachment at low Capillary and Bond numbers using Volume of Fluid and Level Set methods. Chemical Engineering Science, 90, 77 (2013). 